\title{
ESPECTROSCOPIA VIBRACIONAL: SISTEMÁTICA PARA O CÁLCULO DOS ESTIRAMENTOS CO DE COMPLEXOS CARBONÍLICOS E DETERMINAÇÃO DA SUA ATIVIDADE IV E RAMAN
}

\author{
Gelson Manzoni de Oliveira \\ Departamento de Química, Universidade Federal de Santa Maria, 97105-900 Santa Maria - RS
}

Recebido em 11/6/01; aceito em 5/12/01

\begin{abstract}
VIBRATIONAL SPECTROSCOPY: PROCEDURES FOR THE CALCULATION OF THE CO STRETCHINGS OF CARBONYL COMPLEXES AND DETERMINATION OF THEIR IR AND RAMAN ACTIVITY. IR bands related to M-C stretchings are not diagnostically significant for the identification of carbonyl groups in the spectra of carbonyl complexes. Otherwise, the frequency, intensity and number of bands for the CO stretchings provide very useful informations about the number of CO ligands and many others structural proprieties, like the presence of bridged CO groups. We report about a relatively simple and useful method for the determination of the $\mathrm{CO}$ stretchings of carbonyl complexes, which considers only the bond stretching internal coordinates of the CO groups.
\end{abstract}

Keywords: infrared spectra of carbonyl complexes; $\mathrm{CO}$ stretching bands; bond stretching coordinates.

\section{INTRODUÇÃO}

Este estudo é dirigido principalmente a estudantes e pesquisadores que utilizam a espectroscopia no infravermelho como ferramenta para a análise de complexos carbonílicos. Por razões de espaço, não pode ser abrangente a ponto de prescindir dos conhecimentos prévios relacionados com os fundamentos deste método. Assim, pressupõe a familiaridade do leitor com os Grupos Pontuais de moléculas e com as matrizes transformacionais dos vetores dos graus de liberdade (Translacionais, Rotacionais e Vibracionais) para o cálculo das representações reduzíveis e irreduzíveis, tanto destes vetores como da soma total dos graus de liberdade para uma dada molécula. Presume-se também que o leitor saiba deduzir e utilizar as tábuas de caracteres dos grupos pontuais. O embasamento teórico sobre as coordenadas internas é também indispensável, bem como o uso da fórmula de redução para o cálculo das representações irreduzíveis (espécies de simetria) dos estiramentos $\mathrm{CO}$.

Alguns destes assuntos serão abordados, com maior ou menor profundidade, nas próximas seções.

\section{CONSIDERAÇÕES GERAIS}

A utilização mais importante de espectros no infravermelho no laboratório inorgânico está provavelmente relacionada com o reconhecimento de estruturas e o esclarecimento de ligações de complexos carbonílicos e seus derivados. Neste contexto a interpretação de espectros é simplificada quando se analisa os estiramentos $\mathrm{CO}$ e não os estiramentos M-C, das ligações M-CO. Os primeiros produzem bandas intensas e definidas, as quais aparecem bem separadas do restante das vibrações fundamentais da molécula. Ao contrário, as bandas correspondentes aos estiramentos M-C encontram-se na mesma região de outros tipos de vibrações (como das deformações MC-O, por exemplo), tornando-se difícil a sua ordenação. Além disto, vibrações moleculares classificadas como "estiramentos M-C" frequentemente não correspondem a estas formas vibracionais puras ${ }^{1}$.

A derivação da ordem da ligação M-C a partir da localização das bandas $\mathrm{CO}$ baseia-se no pressuposto de uma valência constante para

*e-mail: manzoni@quimica.ufsm.br o átomo de carbono. $\mathrm{O}$ aumento da ordem da ligação $\mathrm{M}-\mathrm{C}$ deve produzir uma diminuição proporcional da ordem da ligação $\mathrm{CO}$, com conseqüente decréscimo da freqüência vibracional do estiramento CO. Por comparação direta das freqüências dos estiramentos $\mathrm{CO}$ de metal-carbonilas e de CO livre obtém-se dados qualitativos importantes: a molécula CO apresenta uma freqüência vibracional de 2143 $\mathrm{cm}^{-1}$. As frequiências dos estiramentos dos grupos CO terminais, em complexos carbonílicos neutros, encontram-se na região entre 2125 - $1850 \mathrm{~cm}^{-1}$, evidenciando uma diminuição da ordem da ligação CO (as freqüências podem cair para valores ainda mais baixos, se forem introduzidas modificações nas carbonilas, capazes de intensificar o sinergismo da ligação M-C).

A presença de pontes $\mathrm{CO}$, duplas ou triplas, pode também ser reconhecida a partir dos espectros no infravermelho. Em concordância com o caráter de dupla ligação, a frequiência vibracional de grupos CO "cetônicos" ( $\mu$-CO, ponte simples) cai para valores situados entre 1750 - $1850 \mathrm{~cm}^{-1}$. Para grupos $\mathrm{CO}$ com função de ponte tripla $\left(\mu_{3}-\mathrm{CO}\right)$ a frequiência dos estiramentos $\mathrm{CO}$ em moléculas neutras oscila entre 1620 e $1730 \mathrm{~cm}^{-1}$.

Além das propriedades acima mencionadas, a Freqüiência, o Número e a Intensidade das bandas permitem também a determinação de simetrias moleculares, a comprovação de isômeros de conformação (confôrmeros), a determinação de ângulos de ligação e o cálculo de constantes de força da ligação carbonílica.

A seguir demonstraremos como se pode calcular, com base em considerações matemáticas e de simetria, o número de estiramentos $\mathrm{CO}$ em espectros no IV para diferentes compostos. Para fins operacionais, a medição de espectros em solução é aconselhada, apesar da interferência do solvente em alguns casos. Os cálculos serão realizados para complexos representativos dos princípios estruturais octaédrico, trigonal bipiramidal e tetraédrico. Ao final, discutiremos brevemente a aplicação do método da simetria local.

\section{CÁLCULOS DOS ESTIRAMENTOS CO}

\section{Metal-Carbonilas com Princípio Estrutural Octaédrico}

$\mathrm{M}(\mathrm{CO})_{6}$

Neste primeiro exemplo abordaremos as duas maneiras de considerar o problema: a análise completa e a forma simplificada, que 
consiste em trabalhar apenas com as coordenadas internas $(\Delta r)$ dos grupos CO. A primeira será tratada resumidamente.

I) Considera-se todos os átomos que constituem a molécula: 13 massas pontuais, 39 vetores ou graus de liberdade $(G)$, e $3 \mathrm{~N}-6=33$ graus de liberdade vibracionais $(V)$. Além disto, 12 coordenadas internas para estiramentos $\mathrm{M}-\mathrm{C}$ e $\mathrm{CO}$ (est.). Inicialmente determina-se a representação reduzível total $\Gamma_{r(G)}$, a representação reduzível de todas as vibrações $\Gamma_{r(V)}$, e a representação reduzível dos estiramentos $\Gamma_{r \text { (est) }}$. A representação reduzível total $\Gamma_{r(G)}$ é o conjunto de caracteres reduzíveis de todas as classes de simetria, e estes podem ser determinados para cada classe de maneira simples mediante uso da equação $\chi_{r(K)}= \pm \mathrm{N}_{R} \cdot(2 \cos \varphi+1)$, derivada da matriz determinante para translações, com sinal positivo para $\boldsymbol{E}$ (Identidade), $\boldsymbol{C}_{\mathbf{n}}$ (eixo rotacional), e negativo para $\boldsymbol{i}$ (inversão), $\sigma$ (plano especular) e $\boldsymbol{S}_{\mathbf{n}}$ (eixo de rotaçãoreflexão); $\mathrm{N}_{R}=$ número de átomos inalterados para a operação de simetria da classe considerada; $\varphi=0^{\circ}$ para $\boldsymbol{E} ; 2 \pi / \mathrm{n}$ para $\boldsymbol{C}_{\mathbf{n}} ; \pi\left(180^{\circ}\right)$ para $\sigma ; 2 \pi$ para $i$, e $2 \pi / \mathrm{n}+\pi$ para $S_{\mathrm{n}}$. A seguir ilustraremos o cálculo dos caracteres reduzíveis das classes $C_{4}\left\{\chi_{r}\left(C_{4}\right)\right\}$ e $S_{4}\left\{\chi_{r}\left(S_{4}\right)\right\}$ para a molécula octaédrica $\mathrm{M}(\mathrm{CO})_{6}$. A Figura 1 apresenta os três eixos rotacionais $C_{4}$ para um complexo hexacarbonílico, os três eixos conjugados contêm os seis elementos de simetria da classe: $C_{4}$ e $C_{4}^{3}$ por eixo $\left(C_{4}^{2}=C_{2} ; C_{4}^{4}=E\right.$, Identidade $)$. O número de átomos cuja posição no espaço não sofre modificação quando se opera qualquer um dos três eixos $C_{4}$ (giro de $90^{\circ}$ ) é igual a 5 , dois grupos lineares $\mathrm{CO}$ e o átomo central. O caráter reduzível de $C_{4}, \chi_{r}\left(C_{4}\right)$, é igual a:

$\chi_{r}\left(C_{4}\right)=\mathrm{N}_{R^{*}} \cdot(2 \cos \varphi+1)=5 \cdot\left(2 \cos 90^{\circ}+1\right)=5$.

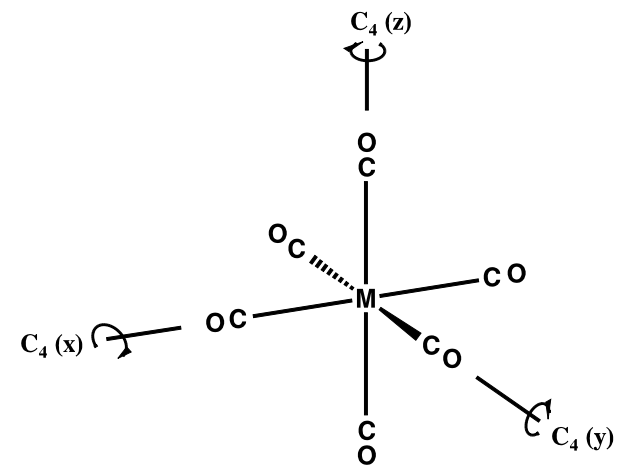

Figura 1. Os três eixos rotacionais $C_{4}$ na molécula octaédrica $\mathrm{M}(\mathrm{CO})_{6}$

Os três eixos de rotação-reflexão formadores da classe $S_{4}$ coincidem com os três eixos rotacionais $C_{4}$ para moléculas octaédricas ( $\mathrm{Fi}$ gura 1), e contêm os seis elementos de simetria da classe, $S_{4}$ e $S_{4}^{3}$ por eixo $\left(S_{4}^{2}=C_{2} ; S_{4}^{4}=E\right.$, Identidade). Ao se executar a operação de simetria $S_{4}$ (giro de $90^{\circ}$ seguido de reflexão no plano central da molécula) em qualquer um dos três eixos $S_{4}$, apenas um átomo - o átomo central - não modifica sua posição. O caráter reduzível da classe $S_{4}$ será o caráter reduzível para qualquer um dos três eixos conjugados:

$\chi_{r}\left(S_{4}\right)=-\mathrm{N}_{R} \cdot(2 \cos \varphi+1)=-1 \cdot\left(2 \cos 270^{\circ}+1\right)=-1$.

A representação $\Gamma_{r(V)}$ é calculada diminuindo-se de $\Gamma_{r(G)}$ a representação reduzível das Translações $\Gamma_{r(T)}$, mais a representação reduzível das Rotações $\Gamma_{r(R)}$ (igual à soma dos caracteres irreduzíveis das espécies em que aparecem translações e rotações, para cada classe de simetria, na tábua de caracteres do grupo pontual $O_{h}$ ). A representação reduzível dos estiramentos $\Gamma_{r \text { (est.) }}$ é calculada operando-se os elementos de simetria do grupo pontual às 12 coordenadas inter$\operatorname{nas}^{2} \Delta r$ (variação de comprimentos de ligação) para os estiramentos
M-C e CO (a representação reduzível para uma determinada classe é o número de coordenadas internas inalteradas). A representação reduzível das deformações (M-CO, C-M-C), $\Gamma_{r \text { (def.) }}$, não será aqui considerada, mas pode ser calculada diminuindo $\Gamma_{r \text { (est.) }}^{r \text { (def.) }} \Gamma_{r(V)}$, método mais eficiente do que utilizar as coordenadas internas $\Delta \alpha$, relacionadas com a variação dos ângulos ${ }^{2}$ de ligação. Conforme se nota, neste primeiro tratamento foram incluídas também as 6 coordenadas $\Delta r$ para os estiramentos M-C. Obtém-se os dados tabelados abaixo, para $\Gamma_{r(G)}, \Gamma_{r(V)}, \Gamma_{r(\text { est. })}$ e $\Gamma_{r(\text { def. })}$.

\begin{tabular}{l|cccccccccc}
$\boldsymbol{O}_{\boldsymbol{h}}$ & $E$ & $8 C_{3}$ & $6 C_{2}$ & $6 C_{4}$ & $3 C_{2}$ & $i$ & $6 S_{4}$ & $8 S_{6}$ & $3 \sigma_{h}$ & $6 \sigma_{d}$ \\
\hline$\Gamma_{r(G)}$ & 39 & 0 & -1 & 5 & -5 & -3 & -1 & 0 & 9 & 5 \\
$\Gamma_{r(V)}$ & 33 & 0 & 1 & 3 & -3 & -3 & -1 & 0 & 9 & 5 \\
$\Gamma_{r(\text { (est. })}$ & 12 & 0 & 0 & 4 & 4 & 0 & 0 & 0 & 8 & 4 \\
$\Gamma_{r \text { (def.) }}$ & 21 & 0 & 1 & -1 & -7 & -3 & -1 & 0 & 1 & 1
\end{tabular}

Neste ponto, o procedimento mais rápido consiste em reduzir $\Gamma_{r(V)}$ e $\Gamma_{r \text { (est.) }}$, determinando-se a representação irreduzível (espécies) dos graus de liberdade vibracionais, $\Gamma_{i(V)}$, e dos estiramentos somente, $\Gamma_{i(\text { est.) }}$. A representação irreduzível das deformações, $\Gamma_{i(\text { def.) }}$, encontra-se pela diferença: $\Gamma_{i(\text { def.) }}=\Gamma_{i(V)}-\Gamma_{i \text { (est.) }}$. Estas representações, identificadas pela simbologia de Mulliken, informam sobre o número de estiramentos e deformações possíveis na molécula e sobre a atividade destas vibrações no infravermelho ou Raman. Este último dado (atividade IV e Raman), é obtido na 3⿳ㅗ e na 4⿳亠丷a colunas da tábua de caracteres: espécies ativas no infravermelho são aquelas às quais pertencem os vetores translacionais $T_{x}, T_{y}$ ou $T_{z}$, uma vez que os componentes $x, y, \mathrm{e} z$ do vetor momento dipolar transformam como translações (terceira coluna). Espécies com atividade Raman são as que apresentam, na quarta coluna da tábua de caracteres, os dados sobre variações da elipsóide de polarizabilidade, ou seja, os produtos dos vetores translacionais.

\{A atividade de uma vibração no infravermelho está condicionada à variação do momento dipolar $(\mu)$, o qual deve necessariamente existir, seja no estado fundamental da molécula ou no estado vibracional excitado; para que um modo vibracional seja Ramanativo é necessária a existência de um momento dipolar induzido pelo campo elétrico da radiação eletromagnética. Este momento dipolar induzido, dividido pelo campo elétrico causador do mesmo, é o tensor polarizabilidade $\alpha$, que pode ser interpretado como uma deformação (mais ou menos intensa) da nuvem de elétrons, pelo componente elétrico da radiação. Para que uma vibração seja Raman-ativa, deve produzir uma modificação da polarizabilidade da molécula\}.

A redução de caracteres (reduzíveis) pode ser feita utilizando-se a fórmula de redução:

$a_{m}=\frac{1}{h} \sum_{K} n \cdot \chi_{i m}(K) \cdot \chi_{r}(K)$,

onde: $a_{m}=\mathrm{n}^{\mathrm{o}}$ de graus de liberdade da espécie $m$ para a qual se está efetuando a redução; $h=$ ordem do grupo pontual, ou número total de elementos de simetria; $\sum_{\mathrm{x}}=$ somatório sobre todas as classes ("soma em linha"); $n=\mathrm{n}^{\mathrm{o}}$ de elementos por classe; $\chi_{i m}(K)=$ caráter irreduzível da espécie $m$ e classe $K ; \chi_{r}(K)=$ caráter reduzível da classe $K$. Um exemplo do uso desta fórmula será dado no ítem II.

Uma outra opção de resolução nesta primeira abordagem consiste em reduzir inicialmente a representação reduzível $\Gamma_{r(G)}$ da tabela anterior (em vez de $\Gamma_{r(V)}$ ), obtendo-se a representação irreduzível $\Gamma_{i(G)}$ para todos os graus de liberdade da molécula $\mathrm{M}(\mathrm{CO})_{6}$ :

$\Gamma_{i(G)}=2 A_{1 g}+2 E_{g}+2 T_{1 g}+2 T_{2 g}+5 T_{1 u}+2 T_{2 u}$ (39 G. L.) 
Da tábua de caracteres do grupo pontual obtém-se diretamente a representação irreduzível das translações e das rotações: $\Gamma_{i(T)}=T_{1 g}$; $\Gamma_{i(R)}=T_{1 u}$ (total = 6 G. L.).

A representação irreduzível de todas as vibrações, $\Gamma_{i(V)}$, é obtida diminuindo-se $\Gamma_{i(T)}$ e $\Gamma_{i(R)}$ de $\Gamma_{i(G)}: \Gamma_{i(V)}=2 A_{1 g}+2 E_{g}+T_{1 g}+2 T_{2 g}+4 T_{1 u}$ $+2 T_{2 u}$ (33 G. L.).

Da tábua de caracteres para o grupo pontual $O_{h}$ (terceira e quarta colunas) obtém-se os dados sobre a atividade das espécies no infravermelho e Raman:

$\Gamma_{i(V)}(\mathrm{IV})=4 T_{1 u} ; \Gamma_{i(V)}(\mathrm{RA})=2 A_{1 g}+2 E_{g}+2 T_{2 g}$

$\Gamma_{i(V)}(\mathrm{IA})=T_{1 g}+2 T_{2 u}(\mathrm{IA}=\mathrm{IV}$ e RA $)$

A representação irreduzível dos estiramentos $\Gamma_{i(\text { est.) }}$, obtida por redução de $\Gamma_{r \text { (est.) }}$, é:

$\Gamma_{i(\text { est. })}=2 A_{1 g}+E_{g}+2 T_{1 u} ; \Gamma_{i(\text { est.) }}(\mathrm{IV})=2 T_{1 u} ; \Gamma_{i \text { (est.) }}(\mathrm{RA})=2 A_{1 g}+E_{g}$

As representações irreduzíveis das deformações são obtidas subtraindo-se $\Gamma_{i(\text { est. })}$ de $\Gamma_{i(V)}$ :

$\Gamma_{i(\text { def. })}=\Gamma_{i(V)}-\Gamma_{i(\text { est. })}=E_{g}+T_{1 g}+2 T_{2 g}+2 T_{1 u}+2 T_{2 u}$

$\Gamma_{i(\text { def. })}(\mathrm{IV})=2 T_{1 u} ; \Gamma_{i(\text { def. })}(\mathrm{RA})=E_{g}+2 T_{2 g}$.

\{O símbolo principal - letra maiúscula - da simbologia de Mulliken para a classificação das espécies - também denominadas raças - de simetria informa o grau de degeneração, ou a dimensão da matriz irreduzível. Caracteres irreduzíveis unidimensionais são identificados pelas letras A e B, caracteres bidimensionais pela letra $\mathrm{E}$, tridimensionais pela letra $\mathrm{T}$ (também $\mathrm{F}$ ), tetra e pentadimensionais pelas letras $\mathrm{G} \mathrm{e} \mathrm{H}$. As letras A e B diferenciam vetores simétricos (1; A) de anti-simétricos $(-1$; B), em relação ao eixo rotacional principal, $C$. Para moléculas com centro de inversão, as notações "g" e "u" (sub-índices) identificam os caracteres irreduzíveis dos vetores simétricos a $i$ (g; a inversão do vetor não inverte o seu sinal) ou antisimétricos (u), por exemplo, $A_{g}, B_{u}, T_{g}$. Quando existe um plano especular horizontal, classifica-se o comportamento simétrico em relação a $\sigma_{h}$ por " ", ", e anti-simétrico por " " " ( $A$ ', $A$ ", etc.). Finalmente, os sub-índices 1 e 2 são notações para o eixo duplo secundário (por exemplo, nos grupos diédricos) ou para planos especulares verticais: para caracteres simétricos em relação a $C_{2}$ usa-se 1, para antisimétricos, 2 (exemplos, $A_{1}, B_{2}$ ). Uma dada propriedade molecular com caráter irreduzível unidimensional, anti-simétrica em relação a $C_{n}$, simétrica com relação à inversão, anti-simétrica à operação $\sigma_{\mathrm{h}}, \mathrm{e}$ simétrica com relação ao eixo duplo secundário ou ao plano vertical, apresentará os caracteres irreduzíveis $-1,1,-1,1$, para as classes $C_{n}$, $i, \sigma_{h}$ e $C_{2}$, respectivamente, e pertencerá à espécie $\left.B_{1 \mathrm{~g}}\right\}$.

Hexacarbonilas metálicas como $\mathrm{Cr}(\mathrm{CO})_{6}$, por exemplo, mostram no espectro de infravermelho ${ }^{3}$ dois estiramentos da espécie $T_{1 u}$ (tripla degeneração), em 2000 ( $v(C O))$ e $441 \mathrm{~cm}^{-1}$ ( $\left.v(\mathrm{MC})\right)$, e duas deformações também pertencentes à espécie $T_{1 u}$, situadas em 668 $(\delta(\mathrm{MCO}))$ e $98 \mathrm{~cm}^{-1}(\delta(\mathrm{CMC}))$.

II) Esta forma alternativa de cálculo (simplificada) restringe-se apenas aos estiramentos CO, sem considerar os demais estiramentos e/ou deformações da molécula. Em conseqüência, dispensa os cálculos anteriores, já que analisa os efeitos dos operadores do grupo pontual da molécula somente sobre as coordenadas internas $\Delta r$ dos estiramentos $C O$. Para uma determinada classe de simetria, o caráter reduzível é o número de coordenadas $\Delta r$ cujas posições não se modificam quando da realização da operação (o que, por sua vez, corresponde ao caráter reduzível da respectiva matriz). Operando-se as diferentes classes de simetria do grupo pontual $\boldsymbol{O}_{h}$ sobre as seis coordenadas internas $\Delta r-\mathrm{CO}\left(\Delta r_{1}, \Delta r_{2}, \Delta r_{3}, \Delta r_{4}, \Delta r_{5}, \Delta r_{6}\right)$ obtém-se os caracteres reduzíveis dos estiramentos $\mathrm{CO}$. As coordenadas internas $\Delta r$-CO localizam-se nas ligações C-O, portanto o número de coordenadas $\Delta r$ inalteradas corresponde ao número de carbonilas cuja posição não se modifica para uma determinada operação de simetria da molécula octaédrica $\mathrm{M}(\mathrm{CO})_{6}$. Para exemplificar, consideremos a representação da molécula $\mathrm{M}(\mathrm{CO})_{6}$ da Figura 2, na qual os números identificam os seis grupos $\mathrm{CO}$ : a operação de qualquer um dos três planos especulares horizontais $\sigma_{h}$ não altera a posição das quatro carbonilas que coincidem com estes planos $(2,3,4,5 ; 1,3,6,5 ; 1,2,6,4)$.

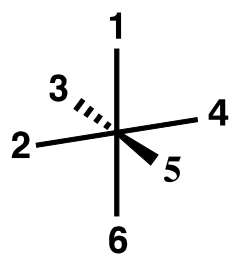

Figura 2. Representação da molécula $M(C O)$

Os caracteres reduzíveis dos estiramentos $\mathrm{CO}$ para as diversas classes de simetria de $\mathrm{M}(\mathrm{CO})_{6}$ são:

\begin{tabular}{l|cccccccccc}
$\boldsymbol{O}_{\boldsymbol{h}}$ & $E$ & $8 C_{3}$ & $6 C_{2}$ & $6 C_{4}$ & $3 C_{2}$ & $i$ & $6 S_{4}$ & $8 S_{6}$ & $3 \sigma_{h}$ & $6 \sigma_{d}$ \\
\hline$\Gamma_{r(\text { est. })}$ & 6 & 0 & 0 & 2 & 2 & 0 & 0 & 0 & 4 & 2
\end{tabular}

Reduzindo-se para todas as espécies do grupo pontual $\boldsymbol{O}_{\boldsymbol{h}}$ determina-se as representações irreduzíveis:

$A_{1 g}=1 / 48[1.1 .6+6.1 .2+3.1 .2+3.1 .4+6.1 .2]=1$

$A_{2 g}=1 / 48[1 \cdot 1 \cdot 6+6 \cdot(-1) \cdot 2+3 \cdot 1 \cdot 2+3 \cdot 1 \cdot 4+6 \cdot(-1) \cdot 2]=0$

$E_{g}=1 / 48[1 \cdot 2 \cdot 6+3 \cdot 2 \cdot 2+3 \cdot 2 \cdot 4]=1$

$T_{1 g}^{g}=1 / 48[1 \cdot 3 \cdot 6+6 \cdot 1 \cdot 2+3 \cdot(-1) \cdot 2+3 \cdot(-1) \cdot 4+6 \cdot(-1) \cdot 2]=0$

$T_{2 g}^{1 g}=1 / 48[1 \cdot 3 \cdot 6+6 \cdot(-1) \cdot 2+3 \cdot(-1) \cdot 2+3 \cdot(-1) \cdot 4+6 \cdot 1 \cdot 2]=0$

$A_{1 u}^{2 g}=1 / 48[1 \cdot 1 \cdot 6+6 \cdot 1 \cdot 2+3 \cdot 1 \cdot 2+3 \cdot(-1) \cdot 4+6 \cdot(-1) \cdot 2]=0$

$A_{2 u}=1 / 48[1 \cdot 1 \cdot 6+6 \cdot(-1) \cdot 2+3 \cdot 1 \cdot 2+3 \cdot(-1) \cdot 4+6 \cdot 1 \cdot 2]=0$

$E_{u}=1 / 48[1.2 .6+3 \cdot 2 \cdot 2+3 \cdot(-2) \cdot 4]=0$

$T_{1 u}=1 / 48[1 \cdot 3 \cdot 6+6 \cdot 1 \cdot 2+3 \cdot(-1) \cdot 2+3 \cdot 1 \cdot 4+6 \cdot 1 \cdot 2]=1$

$T_{2 u}=1 / 48[1 \cdot 3 \cdot 6+6 \cdot(-1) \cdot 2+3 \cdot(-1) \cdot 2+3 \cdot 1 \cdot 4+6 \cdot(-1) \cdot 2]=0$

$$
\Gamma_{i(\mathrm{est} .)}=\Gamma_{i(\mathrm{vCO})}=A_{1 g}+E_{g}+T_{1 u} ; \Gamma_{i(\mathrm{vCO})}(\mathrm{IV})=T_{1 u}
$$

A redução acima foi realizada empregando-se a fórmula abaixo, cujos termos foram identificados no ítem $\mathbf{I}$ :

$a_{m}=\frac{1}{h} \sum_{K} n \cdot \chi_{i m}(K) \cdot \chi_{r}(K)$

Para reduções deste tipo necessita-se dos caracteres irreduzíveis, fornecidos pela tábua de caracteres do grupo pontual. Para fins de complementação, também deste exemplo, reproduzimos na Tabela 1 a tábua de caracteres ${ }^{4}$ do grupo pontual $O_{h}$.

Notar que as espécies encontradas diferem daquelas determinadas anteriormente apenas quanto à quantidade, já que aqui não foram consideradas as coordenadas internas $\Delta r$-M-C.

Espectros no IV de complexos neutros metal-hexacarbonila apresentam uma intensa banda de absorção $v(\mathrm{CO})$ de espécie $T_{1 u}$, situada aproximadamente em $2000 \mathrm{~cm}^{-1}$. Em complexos catiônicos a banda desloca-se para regiões de maior freqüência ${ }^{5}$, em ánions a freqüência de absorção é menor do que $2000 \mathrm{~cm}^{-1}\left\{\mathrm{Mn}(\mathrm{CO})_{6}^{+}: 2090\right.$; $\mathrm{V}(\mathrm{CO})_{6}^{-}$: 1860)\}.

$\mathbf{M}(\mathbf{C O})_{5} \mathbf{X}$; Grupo Pontual $C_{4 v}$

Representação reduzível dos estiramentos $\mathrm{CO}\left(\Gamma_{r(\text { est. })}=\Gamma_{r(\mathrm{vCO})}\right)$ 
Tabela 1. Tábua de caracteres do Grupo Pontual $\boldsymbol{O}_{\boldsymbol{h}}$

\begin{tabular}{l|crrrrrrrrr|r|r}
\hline $\boldsymbol{O}_{\boldsymbol{h}}$ & $E$ & $8 C_{3}$ & $6 C_{2}$ & $6 C_{4}$ & $3 C_{2}$ & $i$ & $6 S_{4}$ & $8 S_{6}$ & $3 \sigma_{h}$ & $6 \sigma_{d}$ & \\
\hline$A_{1 g}$ & 1 & 1 & 1 & 1 & 1 & 1 & 1 & 1 & 1 & 1 & \\
$A_{2 g}$ & 1 & 1 & -1 & -1 & 1 & 1 & -1 & 1 & 1 & -1 & $\left(\alpha_{\left.x^{2}+y^{2}+z^{2}\right)}\right.$ \\
$E_{g}$ & 2 & -1 & 0 & 0 & 2 & 2 & 0 & -1 & 2 & 0 & $\left(R_{x}, R_{y}, R_{z}\right)$ \\
$T_{1 g}$ & 3 & 0 & -1 & 1 & -1 & 3 & 1 & 0 & -1 & -1 & $\left(\alpha_{2 z^{2}-x^{2}-y^{2},}, \alpha_{x^{2}-y^{2}}\right)$ \\
$T_{2 g}$ & 3 & 0 & 1 & -1 & -1 & 3 & -1 & 0 & -1 & 1 & $\left(\alpha_{x z}, \alpha_{y z}, \alpha_{x y}\right)$ \\
$A_{1 u}$ & 1 & 1 & 1 & 1 & 1 & -1 & -1 & -1 & -1 & -1 & \\
$A_{2 u}$ & 1 & 1 & -1 & -1 & 1 & -1 & 1 & -1 & -1 & 1 & \\
$E_{u}$ & 2 & -1 & 0 & 0 & 2 & -2 & 0 & 1 & -2 & 0 & \\
$T_{1 u}$ & 3 & 0 & -1 & 1 & -1 & -3 & -1 & 0 & 1 & 1 & $\left(T_{x}, T_{y}, T_{z}\right)$ \\
$T_{2 u}$ & 3 & 0 & 1 & -1 & -1 & -3 & 1 & 0 & 1 & -1 & \\
\hline
\end{tabular}

\begin{tabular}{l|ccccc}
$\boldsymbol{C}_{4 v}$ & $E$ & $2 C_{4}$ & $C_{2}$ & $2 \sigma_{v}$ & $2 \sigma_{d}$ \\
\hline$\Gamma_{r(\mathrm{vCO})}$ & 5 & 1 & 1 & 3 & 1
\end{tabular}

Representação irreduzível $\Gamma_{i(\mathrm{vCO})}$ (fórmula de redução):

$\Gamma_{i(\mathrm{vCO})}=2 A_{1}+B_{1}+E$

$\Gamma_{i(\mathrm{vCO})}(\mathrm{IV})=2 A_{1}+E$

Carbonilas do tipo $\mathrm{M}(\mathrm{CO})_{5} \mathrm{X}$ mostram no espectro de infravermelho três $v(\mathrm{CO})$ pertencentes às espécies $2 A_{1}+E$. A frequiência da banda correspondente ao estiramento da carbonila axial (da espécie $A_{1}$ ), depende da acidez $\pi$ do ligante não carbonílico: para dois ligantes $\mathrm{X}_{\mathrm{A}}$ e $\mathrm{X}_{\mathrm{B}}$, aquele com caráter $\pi$ ácido mais pronunciado enfraquecerá mais a ligação M-C da carbonila oposta (trans), diminuindo proporcionalmente o comprimento da ligação $\mathrm{CO}$ (aumentando a ordem da ligação), deslocando a banda deste estiramento para maiores frequiências, em relação ao ligante com menor caráter $\pi$ ácido. No entanto, devido à máxima acidez $\pi$ dos grupos $\mathrm{CO}$ - outros ligantes $\pi$ ácidos não competem com tanta intensidade ${ }^{6,7}$ pela densidade eletrônica para a retro-ligação $\pi$ - as bandas $v(\mathrm{CO})$ de carbonilas em posição trans entre si sempre ocorrerão a freqüências maiores do que aquelas de carbonilas semelhantes à carbonila axial de $\mathrm{M}(\mathrm{CO})_{5} \mathrm{X}$ \{ a substituição de um grupo $\mathrm{CO}$ por um ligante $\mathrm{PPh}_{3}$, por exemplo, causa diminuição do comprimento da ligação $\mathrm{M}-\mathrm{C}$ da carbonila situada em posição trans ao novo ligante (o contrário do exemplo acima, para os dois ligantes $\mathrm{X}_{\mathrm{A}}$ e $\mathrm{X}_{\mathrm{B}}$ ); o consequente aumento da densidade eletrônica no átomo de carbono reforça o predomínio da forma de ressonância:

$$
\mathrm{M}=\mathrm{C}=\mathbf{0}
$$

sobre a forma polar

\section{$\overline{\mathbf{M}}-\stackrel{+}{\mathbf{C}} \equiv$ OI}

da ligação M-CO. A correspondente diminuição da constante de força da ligação CO reduz a frequiência do estiramento, com deslocamento para menor freqüência da respectiva banda ${ }^{2,8}$. Em outros compostos carbonílicos estes efeitos são bastante conhecidos e devem ser considerados para a ordenação das bandas: a tendência do oxigênio carbonílico a atrair elétrons $\left(\mathrm{R}_{2} \mathrm{C}=\mathrm{O} \leftrightarrow \mathrm{R}_{2} \mathrm{C}^{+}-\mathrm{O}^{-}\right)$causa o enfraquecimento da constante de força da ligação $\mathrm{C}=\mathrm{O}$ e diminuição da freqüência de absorção da carbonila; grupos eletronegativos ligados ao átomo de carbono competem com o oxigênio por elétrons, diminuindo a contribuição da forma de ressonância polar $\mathrm{C}^{+}-\mathrm{O}^{-}$e aumentando a freqüência de absorção ${ }^{2,9}$. Por exemplo, cetonas $\left(\mathrm{R}_{2} \mathrm{C}=\mathrm{O}\right)$ absorvem em torno de $1715 \mathrm{~cm}^{-1}$, enquanto cloretos de acila $(\mathrm{RClC}=\mathrm{O})$ absorvem na região de $\left.1800 \mathrm{~cm}^{-1}\right\}$.
Nos exemplos abaixo, e nos demais exemplos, as letras a e e estão para axial e equatorial (configurações dos ligantes CO). Os símbolos: w (weak), m (middle), s (strong) e vs (very strong) referem-se à intensidade das bandas. Os estiramentos estão identificados pelas espécies às quais pertencem, juntamente com o dado de configuração, entre parênteses, quando for o caso, e a frequiência é dada em $\mathrm{cm}^{-1}$.

$\mathrm{Mo}(\mathrm{CO})_{5} \mathrm{CH}_{3} \mathrm{CN}: 2125$, w, $\boldsymbol{A}_{\mathbf{1}}(\mathbf{e}) ; 2044$, vs, $\boldsymbol{E} ; 2003, \mathrm{~m}, \boldsymbol{A}_{\mathbf{1}}$ (a).

$\mathrm{V}(\mathrm{CO})_{5} \mathrm{NO}: 2108$, w, $\boldsymbol{A}_{\mathbf{1}}(\mathbf{e}) ; 2064, \mathrm{~m}, \boldsymbol{A}_{\mathbf{1}}(\mathbf{a}) ; 1992$, vs $(\boldsymbol{E})$.

\section{$\mathrm{M}(\mathrm{CO})_{4} \mathbf{X}_{2}$}

(i) trans- $\mathrm{M}(\mathrm{CO})_{4} \mathrm{X}_{2}$; Grupo Pontual $D_{4 h}$

\begin{tabular}{l|cccccccccc}
$\boldsymbol{D}_{4 \boldsymbol{h}}$ & $E$ & $2 C_{4}$ & $C_{2}$ & $2 \mathrm{C}_{2}^{\prime}$ & $2 \mathrm{C}_{2}^{\prime \prime}$ & $i$ & $2 S_{4}$ & $\sigma_{h}$ & $2 \sigma_{v}$ & $2 \sigma_{d}$ \\
\hline$\Gamma_{r(\mathrm{VCO})}$ & 4 & 0 & 0 & 2 & 0 & 0 & 0 & 4 & 2 & 0
\end{tabular}

$\Gamma_{i(\mathrm{vCO})}=A_{1 g}+B_{1 g}+E_{u}$

$\Gamma_{i(\mathrm{vCO})}^{(\mathrm{IV})}=E_{u}$

No espectro de IV observa-se apenas um estiramento IV-ativo da espécie $E_{u}$.

Exemplo: trans- $(\mathrm{CO})_{4} \mathrm{Mo}\left(\mathrm{PPh}_{3}\right)_{2}: v(\mathrm{CO})=1887 \mathrm{~cm}^{-1}$.

(ii) cis- $\mathrm{M}(\mathrm{CO})_{4} \mathrm{X}_{2}$; Grupo Pontual $C_{2 v}$

\begin{tabular}{c|cccc}
$C_{2 v}$ & $E$ & $C_{2}$ & $\sigma_{v(x z)}$ & $\sigma_{v^{\prime}(y z)}$ \\
\hline$\Gamma_{r(\mathrm{VCO})}$ & 4 & 0 & 2 & 2
\end{tabular}

$\Gamma_{i(\mathrm{vCO})}=2 A_{1}+B_{1}+B_{2}=\Gamma_{i(\mathrm{vCO})}(\mathrm{IV})$

Metal-carbonilas com esta configuração apresentam quatro bandas das espécies $2 A_{1}+B_{1}+B_{2}$ no espectro de infravermelho, correspondentes aos quatro $v(\mathrm{CO})$. A freqüência do estiramento das carbonilas equatoriais (espécie $A_{1}$ ) depende da acidez $\pi$ dos ligantes $\mathrm{X}_{2}$. O eixo rotacional $C_{2}$ é bissetriz ao ângulo formado pelas ligações $\mathrm{M}-\mathrm{CO}$ das duas carbonilas equatoriais, conforme demonstrado na Figura 3.

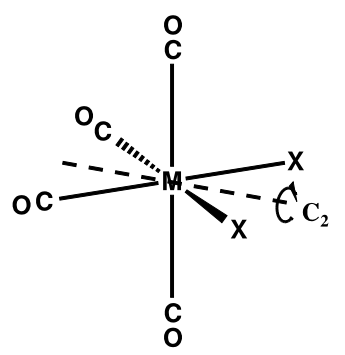

Figura 3. Estrutura de cis- $\mathrm{M}(\mathrm{CO})_{4} \mathrm{X}_{2}$. O eixo $\mathrm{C}_{2}$ é bissetriz ao ângulo formado pelas ligações cis-OC-M-CO 
Exemplos:

Cis-(CO) $)_{4} \mathrm{Mo}\left(\mathrm{PPh}_{3}\right)_{2}: 2014$, w, $\boldsymbol{A}_{1}(\mathbf{a}) ; 1915$, w-m(“ombro"), $\boldsymbol{A}_{1}(\mathbf{e})$; 1900 , vs, $\boldsymbol{B}_{1} ; 1890, \mathrm{~s}, \boldsymbol{B}_{2}$.

Cis-(CO) ${ }_{4}$ Mo bipy : 2015, w-m, $\boldsymbol{A}_{\mathbf{1}}(\mathbf{a}) ; 1906$, vs, $\boldsymbol{B}_{\mathbf{1}}$; 1884, s, $\boldsymbol{A}_{\mathbf{1}}(\mathbf{e})$; $1844, \mathrm{~s}, \boldsymbol{B}_{2}$.

\section{$\mathrm{M}(\mathrm{CO})_{3} \mathbf{X}_{3}$}

(i) $c i s-\mathrm{M}(\mathrm{CO})_{3} \mathrm{X}_{3}$ (facial); Grupo Pontual $C_{3 v}$

\begin{tabular}{l|lcc}
$C_{3 v}$ & $E$ & $2 C_{3}$ & $3 \sigma_{v}$ \\
\hline$\Gamma_{r(v \mathrm{CO})}$ & 3 & 0 & 1
\end{tabular}

$\Gamma_{i(\mathrm{vCO})}=A_{1}+E=\Gamma_{i(\mathrm{vCO})}(\mathrm{IV})$

Observa-se dois $v(\mathrm{CO}) \mathrm{IV}$-ativos pertencentes às espécies $A_{1}+$ $E$. A configuração facial está representada na Figura 4 , o eixo $C_{3}$ coincide com a perspectiva do observador.

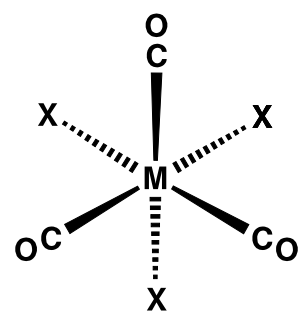

Figura 4. Estrutura de fac- $M(C O)_{3} X_{3}$, ao longo do eixo rotacional $C_{3}$

Exemplo: $\pi-\mathrm{C}_{6} \mathrm{H}_{6} \mathrm{Cr}(\mathrm{CO})_{3}: 1987, \mathrm{~m}, \boldsymbol{A}_{\mathbf{1}} ; 1917$, vs, $\boldsymbol{E}$.

(ii) trans- $\mathrm{M}(\mathrm{CO})_{3} \mathrm{X}_{3}$ (meridional); Grupo Pontual $C_{2 v}$

\begin{tabular}{c|cccc}
$\boldsymbol{C}_{2 v}$ & $E$ & $C_{2}$ & $\sigma_{v(x z)}$ & $\sigma_{v^{\prime}(y z)}$ \\
\hline$\Gamma_{r(\mathrm{vCO})}$ & 3 & 1 & 3 & 1
\end{tabular}

$\Gamma_{i(\mathrm{vCO})}=2 A_{1}+B_{1}=\Gamma_{i(\mathrm{vCO})}(\mathrm{IV})$

Observa-se três $v(\mathrm{CO})$ IV-ativos pertencentes às espécies $2 A_{1}+$ $B_{1}$. A Figura 5 reproduz a configuração meridional, demonstrando também o eixo rotacional $C_{2}$.

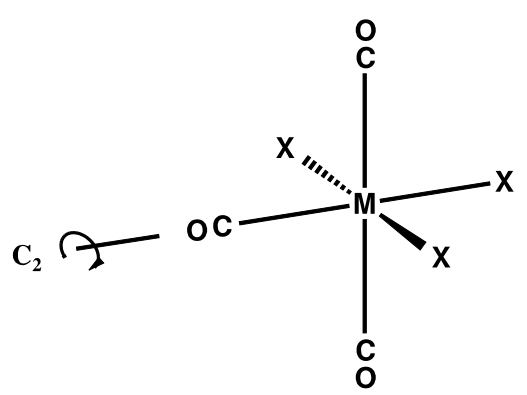

Figura 5. Estrutura de mer-M(CO) ${ }_{3} X_{3}$ e o eixo rotacional $C_{2}$

Exemplo: trans- $(\mathrm{CO})_{3} \mathrm{Mo}\left\{\mathrm{P}(\mathrm{OMe})_{3}\right\}_{3}: 1993, \mathrm{w}, \boldsymbol{A}_{\mathbf{1}} ; 1919, \mathrm{~m}, \boldsymbol{B}_{\mathbf{1}}$; 1890, s, $\boldsymbol{A}_{\mathbf{1}}$.

$\mathrm{M}(\mathrm{CO})_{2} \mathbf{X}_{4}$

(i) cis- $\mathrm{M}(\mathrm{CO})_{2} \mathrm{X}_{4}$; Grupo Pontual $C_{2 v}$

\begin{tabular}{l|lccc}
$\boldsymbol{C}_{2 v}$ & $E$ & $C_{2}$ & $\sigma_{v(x z)}$ & $\sigma_{v^{\prime}(y z)}$ \\
\hline$\Gamma_{r(\mathrm{VCO})}$ & 2 & 0 & 2 & 0
\end{tabular}
$\Gamma_{i(\mathrm{vCO})}=A_{1}+B_{1}=\Gamma_{i(\mathrm{vCO})}(\mathrm{IV})$ $B_{1}$.

Observa-se dois $v(\mathrm{CO}) \mathrm{IV}$-ativos pertencentes às espécies $A_{1}+$ Exemplo: cis-(CO) $)_{2} \mathrm{Mo}\left\{\mathrm{P}(\mathrm{OMe})_{3}\right\}_{4}: 1909, \mathrm{~s}, \boldsymbol{A}_{\mathbf{1}} ; 1856, \mathrm{~s}, \boldsymbol{B}_{\mathbf{1}}$. (ii) trans- $\mathrm{M}(\mathrm{CO})_{2} \mathrm{X}_{4}$; Grupo Pontual $D_{4 h}$

\begin{tabular}{c|cccccccccc}
$\boldsymbol{D}_{4 h}$ & $E$ & $2 C_{4}$ & $C_{2}$ & $2 C_{2}^{\prime}$ & $2 C_{2}^{\prime \prime}$ & $i$ & $2 S_{4}$ & $\sigma_{h}$ & $2 \sigma_{v}$ & $2 \sigma_{d}$ \\
\hline$\Gamma_{r(\mathrm{vCO})}$ & 2 & 2 & 2 & 0 & 0 & 0 & 0 & 0 & 2 & 2
\end{tabular}

$\Gamma_{i(\mathrm{vCO})}=A_{1 g}+A_{2 u}$

$\Gamma_{i(\mathrm{vCO})}(\mathrm{IV})=A_{2 u}$

Observa-se apenas um v(CO) IV-ativo, da espécie $A_{2 u}$. Para complexos desta configuração com ligantes bidentados a simetria pode ser diferente: o complexo trans- $(\mathrm{CO})_{2} \mathrm{Mo}(\mathrm{dpe})_{2}\left(\mathrm{dpe}=\mathrm{H}_{2} \mathrm{PC}_{2} \mathrm{H}_{4} \mathrm{PH}_{2}\right)$, por exemplo, pertence ao grupo pontual $D_{2 h}$ :

\begin{tabular}{l|lccccccc}
$\boldsymbol{D}_{2 \boldsymbol{h}}$ & $E$ & $C_{2(\mathrm{z})}$ & $C_{2(\mathrm{y})}$ & $C_{2(\mathrm{x})}$ & $i$ & $\sigma_{(\mathrm{xy})}$ & $\sigma_{(\mathrm{xz})}$ & $\sigma_{(\mathrm{yz})}$ \\
\hline$\Gamma_{r(\mathrm{vCO})}$ & 2 & 2 & 0 & 0 & 0 & 0 & 2 & 2
\end{tabular}

$\Gamma_{i(\mathrm{vCO})}=A_{g}+B_{1 u}$

$\Gamma_{i(\mathrm{vCO})}(\mathrm{IV})=B_{1 u}$

A banda correspondente ao estiramento de espécie $B_{1 u}$ localizase em $1844 \mathrm{~cm}^{-1}$. A Figura 6 reproduz a estrutura de trans(CO) ${ }_{2} \mathrm{Mo}(\mathrm{dpe})_{2}$.

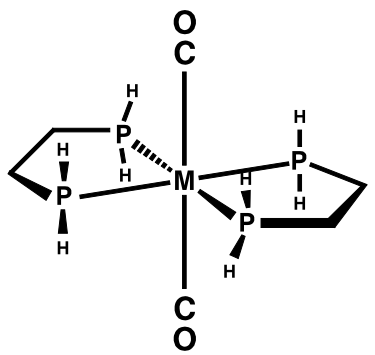

Figura 6. Estrutura de trans- $(\mathrm{CO})_{2} \mathrm{Mo}(\text { dpe })_{2}\left(\right.$ dpe $\left.=\mathrm{H}_{2} \mathrm{PC}_{2} \mathrm{H}_{4} \mathrm{PH}_{2}\right)$

Metal-carbonilas com princípio Estrutural Trigonal Bipiramidal

$\mathbf{M}(\mathbf{C O})_{5} ;$ Grupo Pontual $D_{3 h}$

\begin{tabular}{l|cccccc}
$\boldsymbol{D}_{3 h}$ & $E$ & $2 C_{3}$ & $3 C_{2}$ & $\sigma_{h}$ & $2 S_{3}$ & $3 \sigma_{v}$ \\
\hline$\Gamma_{r(\mathrm{vCO})}$ & 5 & 2 & 1 & 3 & 0 & 3
\end{tabular}

$\Gamma_{i(v \mathrm{CO})}=2 A_{1}^{\prime}+E^{\prime}+A_{2}^{\prime \prime}$
$\Gamma_{i(v \mathrm{CO})}(\mathrm{IV})=A_{2}^{\prime \prime}+E^{\prime}$ E

No espectro de IV são observados dois $v(\mathrm{CO})$, das espécies $A_{2}^{\prime \prime}$ Exemplo: $\left(\mathrm{Fe}(\mathrm{CO})_{5}: 2034, \mathrm{~m}, A_{2}^{\prime \prime} ; 2013\right.$, vs, $E^{\prime}$.

\section{$\mathrm{M}(\mathrm{CO})_{4} \mathrm{X}$}

(i) $\mathbf{X}$ em posição axial; Grupo Pontual $C_{3 v}$

$$
\begin{array}{l|lcc}
C_{3 v} & E & 2 C_{3} & 3 \sigma_{v} \\
\hline \Gamma_{r(\mathrm{vCO})} & 4 & 1 & 2 \\
\Gamma_{i(\mathrm{vCO})}=2 A_{1}+E=\Gamma_{i(\mathrm{vCO})}(\mathrm{IV})
\end{array}
$$


Observa-se três $v(\mathrm{CO}) \mathrm{IV}$-ativos das espécies $2 A_{1}+E$. A frequiência do estiramento da carbonila axial (espécie $A_{1}$ ) depende da acidez $\pi$ do ligante $X$.

Exemplos:

(CO) ${ }_{4} \mathrm{FePPh}_{3}: 2048, \mathrm{~m}, \boldsymbol{A}_{\mathbf{1}}(\mathbf{e}) ; 1974$, w-m, $\boldsymbol{A}_{\mathbf{1}}(\mathbf{a}) ; 1935$, vs, $\boldsymbol{E}$.

(CO) ${ }_{4} \mathrm{FeNH}_{3}$ : 2047, m, $\boldsymbol{A}_{1}(\mathbf{e}) ; 1988$, vs, $\boldsymbol{E}$; 1931, w-m, $\boldsymbol{A}_{\mathbf{1}}$ (a).

(ii) $\mathbf{X}$ em posição equatorial; Grupo Pontual $C_{2 v}$

$$
\begin{array}{l|lccc}
C_{2 v} & E & C_{2} & \sigma_{v(x z)} & \sigma_{v^{\prime}(y z)} \\
\hline \Gamma_{r(v \mathrm{CO})} & 4 & 0 & 2 & 2 \\
\Gamma_{i(\mathrm{vCO})}=2 A_{1}+B_{1}+B_{2}=\Gamma_{i(\mathrm{vCO})}(\mathrm{IV})
\end{array}
$$

Observa-se quatro bandas correspondentes aos quatro $\mathrm{v}(\mathrm{CO}) \mathrm{IV}$ ativos, das espécies $2 A_{1}+B_{1}+B_{2}$.

Exemplo: $(\mathrm{CO})_{4} \mathrm{FeTePh}_{2}(\mathrm{Ph}=$ fenila $): 2066, \mathrm{w}, \boldsymbol{A}_{\mathbf{1}}(\mathbf{a}) ; 2030, \mathrm{~s}, \boldsymbol{A}_{\mathbf{1}}(\mathbf{e})$; 2000, vs, $\boldsymbol{B}_{2} ; 1968, \mathrm{~m}, \boldsymbol{B}_{1}$.

\section{$\mathrm{M}(\mathrm{CO})_{3} \mathbf{X}_{2}$}

(i) Configuração trans ( $\mathbf{X}$ em posição axial); Grupo Pontual $D_{3 h}$

\begin{tabular}{l|lccccc}
$\boldsymbol{D}_{3 h}$ & $E$ & $2 C_{3}$ & $3 C_{2}$ & $\sigma_{h}$ & $2 S_{3}$ & $3 \sigma_{v}$ \\
\hline$\Gamma_{r(\mathrm{~V} \mathrm{CO})}$ & 3 & 0 & 1 & 3 & 0 & 1 \\
$\Gamma_{i(\mathrm{vCO})}=A_{1}^{\prime}+E^{\prime}$ \\
$\Gamma_{i(\mathrm{v} \mathrm{CO})}(\mathrm{IV})=E^{\prime}$
\end{tabular}

Observa-se apenas um v(CO) IV-ativo pertencente à espécie $E^{\prime}$. Exemplo: $(\mathrm{CO})_{3} \mathrm{Fe}\left(\mathrm{PPh}_{3}\right)_{2}: v(\mathrm{CO})=1886 \mathrm{~cm}^{-1}$.

(ii) Configuração cis (X em posição equatorial); Grupo Pontual $C_{2 v}$

\begin{tabular}{c|cccc}
$C_{2 v}$ & $E$ & $C_{2}$ & $\sigma_{v(x z)}$ & $\sigma_{v^{\prime}(y z)}$ \\
\hline$\Gamma_{r(\mathrm{vCO})}$ & 3 & 1 & 1 & 3
\end{tabular}

$\Gamma_{i(\mathrm{vCO})}=2 A_{1}+B_{2}=\Gamma_{i(\mathrm{v} \mathrm{CO})}(\mathrm{IV})$

Observa-se $3 v(\mathrm{CO}) \mathrm{IV}$-ativos pertencentes às espécies $2 A_{1}+B_{2}$. Exemplo: $(\mathrm{CO})_{3} \mathrm{Fe}\left\{\mathrm{P}(\mathrm{OMe})_{3}\right\}_{2}: 2002, \mathrm{w}, \boldsymbol{A}_{\mathbf{1}}(\mathbf{a}) ; 1921, \mathrm{~s}, \boldsymbol{A}_{\mathbf{1}}(\mathbf{e}) ; 1911$, vs, $\boldsymbol{B}_{2}$.

(iii) Configuração cis (X em posição axial e equatorial); Grupo Pontual $C$

$$
\begin{array}{l|lc}
C_{s} & E & \sigma_{h} \\
\hline \Gamma_{r(\mathrm{VCO})} & 3 & 1 \\
\Gamma_{i(\mathrm{VCO})}=2 A^{\prime}+A^{\prime \prime}=\Gamma_{i(\mathrm{VCO})}(\mathrm{IV})
\end{array}
$$

São observados três $v(\mathrm{CO})$ IV-ativos das espécies $2 A^{\prime}+A^{\prime \prime}$. Exemplo: (CO) ${ }_{3} \mathrm{FeC}_{4} \mathrm{H}_{6}$ : 2055, s, $\boldsymbol{A}^{\prime} ; 1989, \mathrm{~s}, \boldsymbol{A}^{\prime} ; 1$ 1979, s, $\boldsymbol{A}^{\prime}$ '.

\section{Metal-carbonilas com princípio Estrutural Tetraédrico}

$\mathbf{M}(\mathbf{C O})_{4} ;$ Grupo Pontual $T_{d}$

\begin{tabular}{l|lcccc}
$\boldsymbol{T}_{\boldsymbol{d}}$ & $E$ & $8 C_{3}$ & $3 C_{2}$ & $6 S_{4}$ & $6 \sigma_{d}$ \\
\hline$\Gamma_{r(\mathrm{vCO})}$ & 4 & 1 & 0 & 0 & 2 \\
$\Gamma_{i(\mathrm{vCO})}=A_{1}+T_{2}$ & \\
$\Gamma_{i(\mathrm{v} \mathrm{CO})}(\mathrm{IV})=T_{2}$
\end{tabular}

Observa-se apenas um estiramento IV-ativo, da espécie $T_{2}$ Exemplos: $\mathrm{Ni}(\mathrm{CO})_{4}: 2060 \mathrm{~cm}^{-1}$, vs; $\left[\mathrm{Co}(\mathrm{CO})_{4}\right]^{-:}: 1890 \mathrm{~cm}^{-1}$, vs; $\left[\mathrm{Fe}(\mathrm{CO})_{4}\right]^{2-:} 1790 \mathrm{~cm}^{-1}$, vs.
Baixos estados de oxidação do átomo central (cargas negativas) favorecem a retro-doação $\pi \mathrm{M} \rightarrow \mathrm{C}$ nos ligantes e o enfraquecimento das ligações $\mathrm{C}-\mathrm{O}$, com conseqüente diminuição da freqüência vibracional dos estiramentos $\mathrm{CO}$. O efeito inverso explica o aumento da freqüência de absorção de complexos carbonílicos catiônicos.

$\mathbf{M}(\mathbf{C O})_{3} \mathbf{X}$; Grupo Pontual $C_{3 v}$

\begin{tabular}{l|ccc}
$C_{3 v}$ & $E$ & $2 C_{3}$ & $3 \sigma_{v}$ \\
\hline$\Gamma_{r(\mathrm{vCO})}$ & 3 & 0 & 1
\end{tabular}

$\Gamma_{i(\mathrm{vCO})}=A_{1}+E=\Gamma_{i(\mathrm{vCO})}(\mathrm{IV})$

Exemplo: $\mathrm{Co}(\mathrm{CO})_{3} \mathrm{NO}: 2108, \mathrm{~m}, \boldsymbol{A}_{\mathbf{1}} ; 2047, \mathrm{vs}, \boldsymbol{E}$.

$\mathbf{M}(\mathbf{C O})_{2} \mathbf{X}_{2} ;$ Grupo Pontual $C_{2 v}$

\begin{tabular}{c|cccc}
$\boldsymbol{C}_{2 v}$ & $E$ & $C_{2}$ & $\sigma_{v(x z)}$ & $\sigma_{v^{\prime}(y z)}$ \\
\hline$\Gamma_{r(v \mathrm{CO})}$ & 2 & 0 & 2 & 0
\end{tabular}

$\Gamma_{i(\mathrm{vCO})}=A_{1}+B_{1}=\Gamma_{i(\mathrm{vCO})}(\mathrm{IV})$

Exemplo: $\mathrm{Fe}(\mathrm{CO})_{2}(\mathrm{NO})_{2}: 2083$, s, $\boldsymbol{A}_{1} ; 2034$, vs, $\boldsymbol{B}_{\mathbf{1}}$.

O decréscimo de simetria na ordem $T_{d} \rightarrow C_{3 v} \rightarrow C_{2 v}$ deve ser acompanhado por um aumento do número de estiramentos, já que os grupos pontuais $C_{3 v}$ e $C_{2 v}$ são subgrupos de $T_{d}$ e as espécies dos subgrupos devem correlacionar-se com as espécies do grupo pontual de origem (maior ordem). A Tabela 2 apresenta a correlação existente entre as representações irreduzíveis $T_{2}$ e $A_{1}$ (IV-inativa) do grupo pontual $T_{d} \mathrm{e}$ aquelas (resultantes) dos subgrupos $C_{3 v}$ e $C_{2 v}$. Nota-se que, enquanto $A_{1}$ se mantém nos dois subgrupos, com modificações nos caracteres da polarizabilidade (com atividade no IV), $T_{2}$ desdobra-se em $A_{1}+E$ (em $C_{3 v}$ ), ocorrendo cisão de $E$ em $B_{1}+B_{2}$, em $C_{2 v}$. O desdobramento de representações irreduzíveis do grupo de origem nas representações irreduzíveis dos subgrupos pode ser determinado com auxílio da fórmula de redução, já vista. Por comparação dos caracteres (dos operadores comuns) do grupo e do correspondente subgrupo, obtém-se, a partir de uma determinada representação irreduzível (conjunto de caracteres irreduzíveis) do grupo de origem, uma representação geralmente reduzível do subgrupo, a qual pode ser decomposta nas repre-

\begin{tabular}{|c|c|c|c|c|}
\hline$T_{d}$ & $\Rightarrow$ & $C_{3 v}$ & $\Rightarrow$ & $C_{2 v}$ \\
\hline$T_{2} \equiv(x, y, z)$ & & $\begin{array}{l}A_{1}-(z) \\
E=(x, y)\end{array}$ & & $\begin{array}{l}A_{1}-(z) \\
B_{1}-(x) \\
B_{2}-(y)\end{array}$ \\
\hline$A_{1}-\left(x^{2}+y^{2}+z^{2}\right)$ & & $A_{1}-\left(x^{2}+y^{2}, z^{2}\right)$ & & $A_{1}-\left(x^{2}, y^{2}, z^{2}\right)$ \\
\hline
\end{tabular}
sentações irreduzíveis correlatas. Estas transformações podem ser obtidas diretamente nas chamadas tabelas de correlação.

Tabela 2. Correlação entre espécies de $\boldsymbol{T}_{d}, C_{3 v}$ e $C_{2 v}$

O decréscimo de simetria $T_{d} \rightarrow C_{3 v}$ ocorre devido à perda de três (dos quatro) eixos $C_{3}$ do grupo pontual $T_{d}$ (desordem trigonal); a perda deste eixo em $C_{3 v}$ causa o decréscimo da sua simetria para $C_{2 v}$. $\mathrm{O}$ grupo pontual $T_{d}$ pode ter sua simetria reduzida diretamente para $C_{2 v}$, quando, devido à denominada desordem rômbica, mantém-se apenas um dos seus três eixos rotacionais duplos \{o grupo pontual $D_{2 d}$ origina-se também de $T_{d}$, por extinção do eixo $S_{4}$ deste (desordem diagonal); as três perturbações (ou desordens) mais comuns no octaedro são: a tetragonal, a trigonal e a rômbica, e os subgrupos pontuais resultantes são $D_{4 h}, D_{3 d}$ e $D_{2 h}$, respectivamente\}. 
Para complexos carbonílicos este princípio (o aumento do número de estiramentos com o decréscimo da simetria) parece apresentar falhas, principalmente no decréscimo (de simetria) de subgrupos entre si $\left(C_{3 v} \rightarrow C_{2 v}\right)$. Isto decorre, primeiramente, do fato de estarmos considerando apenas os estiramentos $\mathrm{CO}$ e não todos os estiramentos da molécula (inclusive os estiramentos M-X). Além disto, o decréscimo de simetria, causado pela substituição de uma carbonila, não é devido apenas a uma alteração da função do ligante, com manutenção da coordenada interna, mas representa também a perda desta coordenada. Mesmo quando se considera apenas os grupos $\mathrm{CO}$, o decréscimo de simetria estará acompanhado de um aumento do número de espécies (estiramentos), se as coordenadas internas $\Delta r$-CO forem mantidas, a nível de simetria local. Este fato pode ser melhor entendido quando se considera o decréscimo da simetria $T_{d} \rightarrow C_{3 v} \rightarrow C_{2 v}$ causado por sucessivas adições aos grupos $\mathrm{CO}$, conforme mostra a Figura 7.

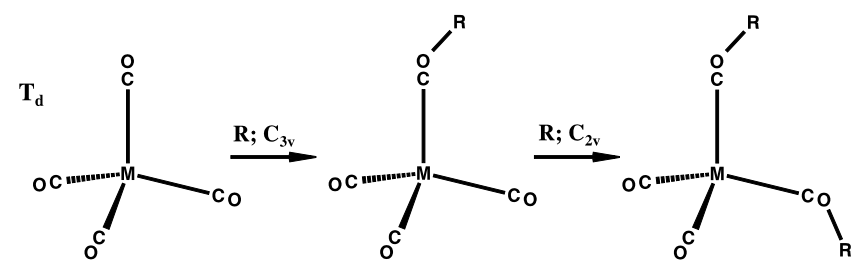

Figura 7. Decréscimo de simetria $T_{d} \rightarrow C_{3 v} \rightarrow C_{2 v}$ por adição de $\boldsymbol{R}$ aos grupos $\mathrm{CO}$. A equivalência entre os ligantes $\mathrm{CO}$ desaparece

Os ligantes COR modificarão as propriedades de simetria da molécula como um todo, porém as propriedades simétricas do conjunto $\mathrm{M}(\mathrm{CO})_{4}$, ou simetria local, serão alteradas apenas devido às diferentes funções exercidas pelos ligantes $\mathrm{CO}$ e COR, os quais deixam de ser equivalentes (a ordem da ligação CO não é mais a mesma para $\mathrm{CO}$ e COR). Isto produz o decréscimo da simetria local $T_{d} \rightarrow$ $C_{3 v} \rightarrow C_{2 v}$, ao mesmo tempo em que as quatro coordenadas internas $\Delta r$-CO (dos ligantes $\mathrm{CO}$ e COR) são mantidas. Nestas condições, a representação irreduzível dos estiramentos $\mathrm{CO}\left(\Gamma_{i(\mathrm{vCO})}\right)$, para o complexo de simetria $C_{3 v}$ da Figura 7, é igual a $2 A_{1}+E$ e para a simetria $C_{2 v}$ da mesma Figura é $2 A_{1}+B_{1}+B_{2}$, resultados de acordo com os dados da Tabela 2.

Quando se considera, além das coordenadas C-O, também as coordenadas internas M-C para o cálculo dos estiramentos dos complexos $\mathrm{MCO}_{3} \mathrm{X}$ e $\mathrm{MCO}_{2} \mathrm{X}_{2}$, obtém-se as seguintes representações irreduzíveis:

Para $\mathrm{MCO}_{3} \mathrm{X}\left(C_{3 v}\right): 2 A_{1}+2 E$ (quatro bandas no espectro de IV para seis estiramentos, já que a espécie $E$ é duplamente degenerada).

Para $\mathrm{MCO}_{2} \mathrm{X}_{2}\left(C_{2 v}\right): 2 A_{1}+2 B_{1}$ (quatro bandas no espectro IV, para quatro estiramentos).

$\{O$ decréscimo de simetria aparece também no estado sólido, quando o grupo espacial cristalográfico apresenta simetria menor do que o "grupo pontual molecular" dos íons componentes. Este fenômeno é denominado simetria local ("site symmetry"). Na sequência discutiremos algumas possibilidades de aplicação deste conceito\}.

\section{Complexos Carbonílicos Polinucleares}

$\mathbf{M n}_{2}(\mathbf{C O})_{10} ;$ Grupo Pontual $D_{4 d}$

\begin{tabular}{l|lcccccc}
$\boldsymbol{D}_{4 d}$ & $E$ & $2 S_{8}$ & $2 C_{4}$ & $2 S_{8}{ }^{3}$ & $C_{2}$ & $4 C_{2}^{\prime}$ & $4 \sigma_{d}$ \\
\hline$\Gamma_{r(\mathrm{vCO})}$ & 10 & 0 & 2 & 0 & 2 & 0 & 4
\end{tabular}

$\Gamma_{i(\mathrm{vCO})}=2 A_{1}+2 B_{2}+E_{1}+E_{2}+E_{3}$

$\Gamma_{i(\mathrm{vCO})}(\mathrm{IV})=2 B_{2}+E_{1}$
No espectro de IV observa-se três bandas correspondentes aos três $v(\mathrm{CO}) \mathrm{IV}$-ativos, das espécies $2 B_{2}+E_{1}$. Exemplo: $\mathrm{Mn}_{2}(\mathrm{CO})_{10}: 2044, \mathrm{w}-\mathrm{m}, \boldsymbol{B}_{2} ; 2013, \mathrm{vs}, \boldsymbol{E}_{\mathbf{1}} ; 1983, \mathrm{~s}, \boldsymbol{B}_{2}$.

A estrutura do complexo binuclear de manganês está representada na Figura 8.

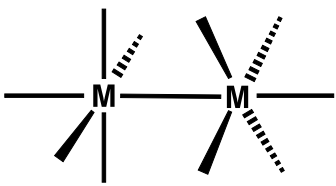

Figura 8. Estrutura de $\mathrm{Mn}_{2}(\mathrm{CO})_{10}$

$\mathrm{Fe}_{\mathbf{2}}(\mathbf{C O})_{9} ;$ Grupo Pontual $D_{3 h}$

Para complexos deste tipo deve-se diferenciar entre grupos $\mathrm{CO}$ terminais $\left\{(\mathrm{CO})_{\mathrm{t}}\right\}$ e pontes $\left\{(\mathrm{CO})_{\mu}\right\}$. É possível obter-se as representações para os dois tipos de estiramentos separadamente, operando-se (em separado) as coordenadas internas para as seis carbonilas terminais e para os três grupos CO ligados aos dois átomos de ferro. A Figura 9 mostra a estrutura do complexo $\mathrm{Fe}_{2}(\mathrm{CO})_{9}$. Na tabela abaixo, a notação $\Gamma_{r(\mathrm{vCO})}$ refere-se à representação reduzível dos estiramentos de todas as carbonilas, terminais e pontes, a qual deve ser igual à soma das representações reduzíveis dos estiramentos das carbonilas terminais $\left(\Gamma_{r(\mathrm{vCO}) t}\right)$ e pontes $\left(\Gamma_{r(\mathrm{vCO}) \mu}\right)$.

\begin{tabular}{l|cccccc}
$\boldsymbol{D}_{3 h}$ & $E$ & $2 C_{3}$ & $3 C_{2}$ & $\sigma_{h}$ & $2 S_{3}$ & $3 \sigma_{v}$ \\
\hline$\Gamma_{r(\mathrm{vCO})}$ & 9 & 0 & 1 & 3 & 0 & 3 \\
$\Gamma_{r(\mathrm{vCO}) t}$ & 6 & 0 & 0 & 0 & 0 & 2 \\
$\Gamma_{r(\mathrm{vCO}) \mu}$ & 3 & 0 & 1 & 3 & 0 & 1
\end{tabular}

$\Gamma_{i(\mathrm{vCO})}=2 A_{1}^{\prime}+2 E^{\prime}+A_{2}^{\prime \prime}+E^{\prime \prime} ; \Gamma_{i(\mathrm{vCO})}(\mathrm{IV})=A_{2}^{\prime \prime}+2 E^{\prime}$

$\Gamma_{i(\mathrm{vCO}) t}=A_{2}^{\prime \prime}+E^{\prime} ; \Gamma_{i(v \mathrm{CO}) \mu}=E^{\prime}$.

Observa-se no espectro de infravermelho três bandas correspondentes aos três $v(\mathrm{CO}) \mathrm{IV}$-ativos: dois $v(\mathrm{CO})$ das carbonilas terminais, de espécies $A_{2}^{\prime \prime}+E^{\prime}$, e um estiramento da espécie $E$, dos grupos $\mathrm{CO}$ exercendo funções de pontes.

Exemplo:

$\mathrm{Fe}_{2}(\mathrm{CO})_{9}$ (em pastilha de $\mathrm{KBr}$ ): 2066, m, $\boldsymbol{A}$ "; 2038, vs, $\boldsymbol{E}$ '; 1855 , 1851, vs, $\boldsymbol{E}^{\prime}$ (banda mais larga, bipartida).

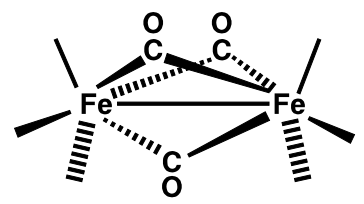

Figura 9. Estrutura de $\mathrm{Fe}_{2}(\mathrm{CO})_{9}$

\section{Introdução ao Método da Simetria Local}

Em virtude do pequeno acoplamento (interação) estrutural existente entre grupos de átomos da mesma molécula, em clusters carbonílicas e complexos carbonílicos/organometálicos (mistos), é possível deduzir e interpretar espectros no infravermelho para estes compostos considerando apenas a simetria de determinadas partes da molécula, ao invés da simetria da molécula como um todo. Este é o princípio do método da simetria local, o qual demonstraremos a seguir para alguns complexos representativos. 
$\mathrm{Os}_{3}(\mathrm{CO})_{12}$

A molécula $\mathrm{Os}_{3}(\mathrm{CO})_{12}$, cuja estrutura encontra-se representada na Figura 10, pertence ao grupo pontual $D_{3 h}$ :

\begin{tabular}{l|cccccc}
$\boldsymbol{D}_{3 h}$ & $E$ & $2 C_{3}$ & $3 C_{2}$ & $\sigma_{h}$ & $2 S_{3}$ & $3 \sigma_{v}$ \\
\hline$\Gamma_{r(\mathrm{VCO})}$ & 12 & 0 & 0 & 6 & 0 & 2
\end{tabular}

$\Gamma_{i(\mathrm{vCO})}=2 A_{1}^{\prime}+A_{2}^{\prime}+3 E^{\prime}+A_{2}^{\prime \prime}+E^{\prime \prime}$

$\Gamma_{i(\mathrm{vCO})}(\mathrm{IV})=A_{2}^{\prime \prime}+3 E^{\prime}$

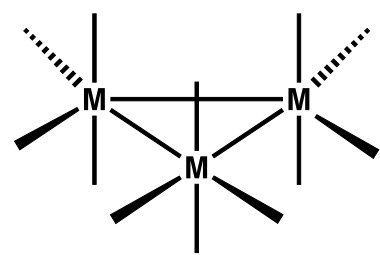

Figura 10. Estrutura de $\mathrm{Os}_{3}(\mathrm{CO})_{12}$. A simetria local nos átomos do metal é $C_{2 v}$

No entanto, o espectro no infravermelho da cluster $\mathrm{Os}_{3}(\mathrm{CO})_{12}$ não corresponde àquele de uma molécula do grupo pontual $D_{3 h}$, significa, as quatro bandas não pertencem às espécies $A_{2}^{\prime \prime}+3 E^{\prime}$. Em vez disto, em razão da simetria $C_{2 v}$ localizada nos grupos $\mathrm{Os}(\mathrm{CO})_{4}$ da molécula de $\mathrm{Os}_{3}(\mathrm{CO})_{12}$ (ver Figura 10), predomina a simetria local $C_{2 v}$, e observa-se um espectro de quatro bandas típico para uma molécula do tipo cis- $\mathrm{M}(\mathrm{CO})_{4} \mathrm{X}_{2}$, deste grupo pontual.

Quando se opera as classes de simetria do grupo pontual $C_{2 v}$ para as coordenadas internas $\Delta r-\mathrm{CO}$ de um grupo $\mathrm{Os}(\mathrm{CO})_{4}$, tem-se:

\begin{tabular}{c|cccc}
$C_{2 v}$ & $E$ & $C_{2}$ & $\sigma_{v(x z)}$ & $\sigma_{v^{\prime}(y z)}$ \\
\hline$\Gamma_{r(v \mathrm{CO})}$ & 4 & 0 & 2 & 2
\end{tabular}

$\Gamma_{i(\mathrm{v} \mathrm{CO})}=2 A_{1}+B_{1}+B_{2}=\Gamma_{i(\mathrm{vCO})}(\mathrm{IV})$

A atribuição das bandas do espectro de infravermelho de $\mathrm{Os}_{3}(\mathrm{CO})_{12}$ às espécies e configurações (axial e equatorial) dos estiramentos, e a correspondência com as espécies dos estiramentos para o grupo pontual $D_{3 h}$, encontram-se resumidas abaixo.

\begin{tabular}{c|c}
$C_{2 v}$ & $D_{3 h}$ \\
$2068 \mathrm{~cm}^{-1}, \mathrm{vs}, \boldsymbol{A}_{1}$ & $E^{\prime}(\mathbf{a})$ \\
$2035 \mathrm{~cm}^{-1}, \mathrm{vs}, \boldsymbol{B}_{\mathbf{1}}$ & $A_{2}^{\prime \prime}(\mathbf{a})$ \\
$2014 \mathrm{~cm}^{-1}, \mathrm{~s}, \boldsymbol{A}_{1}$ & $E^{\prime}(\mathbf{e})$ \\
$2002 \mathrm{~cm}^{-1}, \mathrm{~s}, \boldsymbol{B}_{2}$ & $E^{\prime}(\mathbf{e})$
\end{tabular}

Os espectros no infravermelho ${ }^{10}$ dos complexos $\mathrm{BrMn}(\mathrm{CO})_{5} \mathrm{e}$ $\mathrm{BrMn}(\mathrm{CO})_{4} \mathrm{PPh}_{2} \mathrm{Si}(\mathrm{CH})_{3}$, este obtido por substituição de uma carbonila cis pelo ligante $\mathrm{PPh}_{2} \mathrm{Si}(\mathrm{CH})_{3} \mathrm{em} \mathrm{BrMn}(\mathrm{CO})_{5}$, representam exemplos ilustrativos de aplicação do método da simetria local, dos efeitos da substituição de ligantes com menor caráter $\pi$ ácido sobre a freqüência de absorção dos grupos $\mathrm{CO}$ em posição trans, e das conseqüências do decréscimo de simetria sobre a cisão de espécies: $\mathrm{O}$ complexo $\mathrm{Mn}(\mathrm{CO})_{5} \mathrm{Br}$ pertence ao grupo pontual $C_{4 v}$, os estiramentos $\mathrm{CO}$ devem apresentar um modelo semelhante àqueles dos exemplos anteriormente apresentados para este grupo pontual. Os estiramentos de $\mathrm{Mn}(\mathrm{CO})_{5} \mathrm{Br}$ apresentam as seguintes freqüências: $2137,4 \mathrm{~cm}^{-1}, \mathrm{~m}$, $\boldsymbol{A}_{1}(\mathbf{e}) ; 2045,4 \mathrm{~cm}^{-1}$, vs, $\boldsymbol{E} ; 1990,3 \mathrm{~cm}^{-1}$, s, $\boldsymbol{A}_{1}(\mathbf{a})$. A banda intensa de espécie $E$, situada em $2045,4 \mathrm{~cm}^{-1}$ deve corresponder aos dois modos vibracionais degenerados dos estiramentos "assimétricos" das quatro carbonilas equatoriais, cujo estiramento simétrico, $A_{1}$, localiza-se em $2137,4 \mathrm{~cm}^{-1}$.
Pelo método da simetria local, o retículo parcial $\mathrm{Mn}(\mathrm{CO})_{4}$ do complexo $\mathrm{BrMn}(\mathrm{CO})_{4} \mathrm{PPh}_{2} \mathrm{Si}(\mathrm{CH})_{3}$ pertence ao grupo pontual $C_{2 v}$, deve portanto apresentar um mesmo modelo de absorção no IV para moléculas deste grupo com quatro coordenadas internas, como cis$\mathrm{M}(\mathrm{CO})_{4} \mathrm{X}_{2}$ e a cluster $\mathrm{Os}_{3}(\mathrm{CO})_{12}$. As quatro bandas vCO no espectro de infravermelho de $\mathrm{BrMn}(\mathrm{CO})_{4} \mathrm{PPh}_{2} \mathrm{Si}(\mathrm{CH})_{3}$ apresentam as seguintes localizações e intensidades: 2076,2 $\mathrm{cm}^{-1}, \mathrm{~m} ; 2051 \mathrm{~cm}^{-1}, \mathrm{~m}$; $1994 \mathrm{~cm}^{-1}$, vs; $1952,4 \mathrm{~cm}^{-1}$, vs.

Os estiramentos $\mathrm{CO}$ de $\mathrm{Mn}(\mathrm{CO})_{5} \mathrm{Br}$ e $\mathrm{BrMn}(\mathrm{CO})_{4} \mathrm{PPh}_{2} \mathrm{Si}(\mathrm{CH})_{3}$ pertencem às espécies $2 A_{1}+E$, e $2 A_{1}+B_{1}+B_{2}$, respectivamente. Conforme é mostrado na Tabela 3 (correlação do grupo pontual $C_{4 v}$ e seu subgrupo $C_{2 v}$ ), no decréscimo de simetria de $C_{4 v}$ para $C_{2 v}$ a espécie $\boldsymbol{E}$ desdobra-se em $\boldsymbol{B}_{\mathbf{1}}+\boldsymbol{B}_{\mathbf{2}}$ (como ocorre no decréscimo de simetria $C_{3 v}$ $\left.\rightarrow C_{2 v}\right)$, as quais $\left(B_{1}\right.$ e $\left.B_{2}\right)$ identificam um estiramento axial e um estiramento equatorial, respectivamente. $\mathrm{O}$ estiramento dos quatro grupos $\mathrm{CO}$ de configuração equatorial (trans, entre si) de $\mathrm{Mn}(\mathrm{CO})_{5} \mathrm{Br}$, de espécie $E\left(2045,4 \mathrm{~cm}^{-1}\right)$, desdobra-se, portanto, nos dois estiramentos de espécies $B_{1}$ (a) e $B_{2}(\mathbf{e})$ de $\mathrm{BrMn}(\mathrm{CO})_{4} \mathrm{PPh}_{2} \mathrm{Si}(\mathrm{CH})_{3}$, aos quais podem ser atribuídas as bandas localizadas em $2051 \mathrm{~cm}^{-1}$ (a) e $1952,4 \mathrm{~cm}^{-1}$ (e). Desta forma, a substituição de uma das quatro carbonilas equatoriais em $\mathrm{Mn}(\mathrm{CO})_{5} \mathrm{Br}$, pelo ligante $\mathrm{PPh}_{2} \mathrm{Si}(\mathrm{CH})_{3}$, causou diminuição da frequiência vibracional do estiramento da carbonila trans em relação a este de 2045,4 $\mathrm{cm}^{-1}$ (em $\mathrm{Mn}(\mathrm{CO})_{5} \mathrm{Br}$ ), para 1952,4 $\mathrm{cm}^{-1}$, em $\mathrm{BrMn}(\mathrm{CO})_{4} \mathrm{PPh}_{2} \mathrm{Si}(\mathrm{CH})_{3}$. A espécie $\boldsymbol{A}_{1}(\mathbf{a})$ de $\mathrm{Mn}(\mathrm{CO})_{5} \mathrm{Br}(\mathrm{vCO}-\mathrm{Mn}-$ $\mathrm{Br})$, identifica em $\mathrm{BrMn}(\mathrm{CO})_{4} \mathrm{PPh}_{2} \mathrm{Si}(\mathrm{CH})_{3}$ um dos dois estiramentos das carbonilas axiais deste complexo; a carbonila axial de $\mathrm{Mn}(\mathrm{CO})_{5} \mathrm{Br}$ tem configuração equatorial no sililfosfano-complexo, e seu estiramento pertence à espécie $\boldsymbol{A}_{\mathbf{1}}(\mathbf{e})$. A pequena variação da frequiência deste estiramento nos dois complexos permite concluir que o modelo de deslocamento de bandas de $\mathrm{BrMn}(\mathrm{CO})_{4} \mathrm{PPh}_{2} \mathrm{Si}(\mathrm{CH})_{3}$ é o mesmo da cluster $\mathrm{Os}_{3}(\mathrm{CO})_{12}$.

Tabela 3. Tabela de correlação para os grupos Pontuais $C_{4 v} \rightarrow C_{2 v}$; As espécies $B_{1}$ e $B_{2}$, de $C_{4 v}$, originarão $A_{1}$ ou $A_{2}$, de $C_{2 v}$, conforme sejam considerados os planos especulares $\sigma_{\mathrm{v}}$ ou $\sigma_{\mathrm{d}}$, de $C_{4 v}$.

\begin{tabular}{ccc}
\hline$C_{4 v}$ & $C_{2 v}, \sigma_{v}$ & $C_{2 v}, \sigma_{\mathrm{d}}$ \\
\hline$A_{1}$ & $A_{1}$ & $A_{1}$ \\
$A_{2}$ & $A_{2}$ & $A_{2}$ \\
$B_{1}$ & $A_{1}$ & $A_{2}$ \\
$B_{2}$ & $A_{2}$ & $A_{1}$ \\
$E$ & $B_{1}+B_{2}$ & $B_{1}+B_{2}$ \\
\hline
\end{tabular}

A comparação entre espécies, freqüências, tipos de estiramentos e configurações dos grupos $\mathrm{CO}$, para $\mathrm{Mn}(\mathrm{CO})_{5} \mathrm{Br}$ e $\mathrm{BrMn}(\mathrm{CO})_{4} \mathrm{PPh}_{2} \mathrm{Si}(\mathrm{CH})_{3}$, encontra-se resumida na Tabela 4.

$\eta^{5}-\mathrm{C}_{5} \mathrm{H}_{5} \mathrm{Mn}(\mathrm{CO})_{3}$

$\mathrm{O}$ retículo molecular parcial $\mathbf{M n}(\mathbf{C O})_{3}$ pertence ao grupo pontual $C_{3 v}$ :

\begin{tabular}{l|lcc}
$\boldsymbol{C}_{3 v}$ & $E$ & $2 C_{3}$ & $3 \sigma_{v}$ \\
\hline$\Gamma_{r(v \mathrm{CO})}$ & 3 & 0 & 1
\end{tabular}

$\Gamma_{i(\mathrm{vCO})}=A_{1}+E=\Gamma_{i(\mathrm{vCO})}(\mathrm{IV})$

Exemplo: $\pi-\mathrm{C}_{5} \mathrm{H}_{5} \mathrm{Mn}(\mathrm{CO})_{3}: 2025, \mathrm{~m}, \boldsymbol{A} \mathbf{1} ; 1938, \mathrm{vs}, \boldsymbol{E}$. A estrutura da molécula encontra-se representada na Figura 11.

$\eta^{5}-\mathbf{C}_{5} \mathbf{H}_{5} \mathbf{M}(\mathbf{C O})_{3} \mathbf{X}(\mathbf{M}=\mathrm{Mo}, \mathrm{W})$

(i) $\mathrm{X}=$ halogênio, $\mathrm{SiR}_{3}$ :

Segundo o método da simetria local, o grupo pontual a que pertence o retículo parcial, quadrado piramidal, $\mathbf{M}(\mathbf{C O})_{3} \mathbf{X}$, é $C_{\mathrm{s}}$ : 
Tabela 4. Comparação entre as espécies, configurações dos grupos $\mathrm{CO}$ e tipos de estiramentos para os complexos $\mathrm{BrMn}(\mathrm{CO})_{5}\left(C_{4 v}\right)$ e $\mathrm{BrMn}(\mathrm{CO})_{4} \mathrm{PPh}_{2} \mathrm{Si}\left(\mathrm{CH}_{3}\right)_{3}\left(C_{2 v}\right)$.

\begin{tabular}{|c|c|c|c|c|c|}
\hline \multicolumn{4}{|c|}{$\operatorname{BrMn}(\mathrm{CO})_{5} \quad\left(\boldsymbol{C}_{4 v}\right)$} & \multicolumn{2}{|c|}{$\operatorname{BrMn}(\mathrm{CO})_{4} \mathrm{PPh}_{2} \mathrm{Si}\left(\mathrm{CH}_{3}\right)_{3} \quad\left(C_{2 v}\right)$} \\
\hline Espécie & $\bar{v}\left(\mathrm{~cm}^{-1}\right)$ & $\mathrm{vCO}$ & Espécie & $\bar{v}\left(\mathrm{~cm}^{-1}\right)$ & vCO \\
\hline$A_{1}(\mathrm{e})$ & 2137,4 & 4 grupos $\mathrm{CO}$ equatoriais, trans entre si & $A_{1}(\mathrm{e})$ & 1994 & $\mathrm{CO}-\mathrm{Mn}-\mathrm{Br}$ (trans) \\
\hline$E(\mathrm{e})$ & 2045,4 & idem. & $B_{1}(\mathrm{a})$ & 2051 & CO-Mn-CO (trans) \\
\hline & & & $B_{2}(\mathrm{e})$ & 1952,4 & $\mathrm{CO}-\mathrm{Mn}-\mathrm{PPh}_{2} \mathrm{SiMe}_{3}$ (trans) \\
\hline$A_{1}(\mathrm{a})$ & 1990,3 & $\mathrm{CO}-\mathrm{Mn}-\mathrm{Br}$ (trans) & $A_{1}(\mathrm{a})$ & 2076,2 & $\mathrm{CO}-\mathrm{Mn}-\mathrm{CO}$ (trans) \\
\hline
\end{tabular}

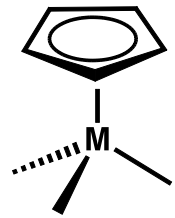

Figura 11. Estrutura de $\mathrm{CpMn}(\mathrm{CO})_{3}$

$$
\begin{aligned}
& \begin{array}{l|ll}
\boldsymbol{C}_{\mathrm{s}} & E & \sigma_{h} \\
\hline \Gamma_{r(\mathrm{vCO})} & 3 & 1
\end{array} \\
& \Gamma_{i(\mathrm{vCO})}=2 A^{\prime}+A^{\prime \prime}=\Gamma_{i(\mathrm{vCO})}(\mathrm{IV})
\end{aligned}
$$

Observa-se no espectro de IV três bandas correspondentes aos três v(CO) IV-ativos das espécies $2 A^{\prime}+A^{\prime \prime}$.

Exemplo:

$\eta^{5}-\mathrm{C}_{5} \mathrm{H}_{5} \mathrm{Mo}(\mathrm{CO})_{3} \mathrm{SiCl}_{3}: 2041, \mathrm{~s}, \boldsymbol{A}$ '; 1976, s, $\boldsymbol{A}^{\prime}$; 1959, s, $\boldsymbol{A}^{\prime}$ '. A Figura 12 reproduz a estrutura do complexo $\eta^{5}-\mathrm{C}_{5} \mathrm{H}_{5} \mathrm{M}(\mathrm{CO})_{3} \mathrm{X}$.

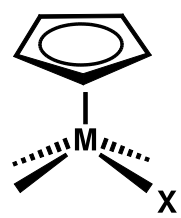

Figura 12. Estrutura de $\operatorname{CpM}(\mathrm{CO})_{3} \mathrm{X} \quad\left(\mathrm{X}=\right.$ Halogênio, $\left.\mathrm{SiR}_{3}\right)$

\section{(ii) $\mathrm{X}=\mathrm{H}$}

Em virtude do seu pequeno tamanho, o ligante hidrido requer bem menos espaço do que os grupos $\mathrm{CO}$, e a simetria local resultante é praticamente $C_{3 v}$. Esta relação está demonstrada na estrutura ideal do hidrido-complexo da Figura 13.

\begin{tabular}{l|ccc}
$C_{3 v}$ & $E$ & $2 C_{3}$ & $3 \sigma_{v}$ \\
\hline$\Gamma_{r(v \mathrm{CO})}$ & 3 & 0 & 1
\end{tabular}

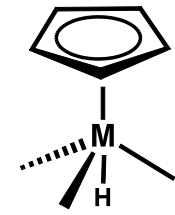

Figura 13. Estrutura ideal de $\mathrm{CpM}(\mathrm{CO})_{3} \mathrm{H}$

$\Gamma_{i(\mathrm{vCO})}=A_{1}+E=\Gamma_{i(\mathrm{vCO})}(\mathrm{IV})$

No espectro de infravermelho observa-se dois estiramentos IVativos de espécies $A_{1}+E$.

Exemplo:

$\eta^{5}-\mathrm{C}_{5} \mathrm{H}_{5} \mathrm{~W}(\mathrm{CO})_{3} \mathrm{H}: 2026, \mathrm{~m}-\mathrm{s}, \boldsymbol{A}_{\mathbf{1}} ; 1935$, vs, $\boldsymbol{E}$. Em razão da troca ativa $\mathrm{H}$ / Halogênio, espectros de IV de hidrido-complexos não devem ser medidos em solventes clorados.

\section{REFERÊNCIAS}

1. Cotton, F. A.; Wilkinson, G. Em Advanced Inorganic Chemistry; John Wiley \& Sons: New York, 4. ed., 1985, p. 1091.

2. Colthup, N. B.; Daly, L. H.; Wiberley, S. E. Em Introduction to Infrared and Raman Spectroscopy; Academic Press: San Diego,3. ed., 1990, p. 491.

3. Lorenz, I.-P. Em Organometallchemie von Übergangsmetallen; Attempto Verlag: Tübingen, 1990, p. 58.

4. Hollas, J. M. Em Die Symmetrie von Molekülen - Eine Einführung in die Anwendung von Symmetriebetrachtungen in der Chemie; de Gruyter, W., ed.; Berlin - New York, 1975, p. 63.

5. Lorenz, I.-P. Em Gruppentheorie und Molekülsymmetrie - Anwendung auf Schwingungs- und Elektronenzustände; Attempto Verlag: Tübingen, 1992, p. 194.

6. Paz-Sandoval, M. A.; Saavedra, P. J.; Villareal, N. Z.; Hoz, M. J.; Nathan, P. J.; Ernst, R. D.; Arif, A. M.; Organometallics 1992, 11, 2467.

7. de Oliveira, G. M.; Hörner, M.; Seiffert, M.; Bortoluzzi A. J.; J. Chem. Crystallogr. 1999, 29, 193.

8. Christen, H. R. Em Grundlagen der Allgemeinen und Anorganischen Chemie; Otto Salle Verlag / Verlag Sauerländer: Frankfurt, 7. ed., 1982.

9. Nakamoto, K. Em Infrared and Raman Spectra of Inorganic and Coordination Compounds, 4. ed., John Wiley \& Sons: New York, 1986.

10. Seiffert, M.; Dissertação de Mestrado, Universidade Federal de Santa Maria, Brasil, 1997. 